\title{
Viewpoint
}

\section{Mapping the luminescence of a single molecule}

\author{
Marina Pivetta \\ Ecole Polytechnique Fédérale de Lausanne (EPFL), Institut de Physique de la Matière Condensée, CH-1015 Lausanne, \\ Switzerland
}

Published November 15, 2010

Spectroscopic mapping of the luminescence of aromatic single molecules reveals the spatial distribution of radiative transitions at submolecular resolution.

Subject Areas: Nanophysics, Materials Science

\author{
A Viewpoint on: \\ Viewing the Interior of a Single Molecule: Vibronically Resolved Photon Imaging at Submolecular Resolution \\ Chi Chen, Ping Chu, C. A. Bobisch, D. L. Mills and W. Ho \\ Phys. Rev. Lett. 105, 217402 (2010) - Published November 15, 2010
}

In the world of nanoscience-at atomic and molecular scales-properties of matter differ from those in everyday objects. Of much current interest are singlemolecule experiments [1-3] that allow us to focus on the influence of the local environment on molecules, while avoiding averaging effects. Different techniques have been developed based on the luminescence of molecules, of which the more interesting ones combine a scanning probe microscopy technique with an optical spectroscopic measurement, such as scanning near-field optical microscopy (SNOM) that can be used for fluorescence or Raman spectroscopy, or tip-enhanced Raman spectroscopy (TERS). These techniques detect single molecules-with a spatial resolution of a few tens of nanometers in the best cases-but this resolution is not good enough to probe the luminescence of a molecule at the submolecular scale.

Molecules in the spectroscopy setups described above are excited by incoming light. Electrons that tunnel between the tip and the specimen in a scanning tunneling microscope (STM) constitute yet another source of excitation-a kind of electroluminescence experiment [Fig. 1(a)]. Now, in a paper appearing in Physical Review Letters, Chi Chen and colleagues at the University of California, Irvine, describe how they used tunneling current as an excitation source to obtain fluorescence images of individual molecules of porphyrin [4]. Porphyrins-aromatic molecules composed of four pyrrole $\left(\mathrm{C}_{4} \mathrm{H}_{4} \mathrm{NH}\right)$ rings in a plane-can bind a metal atom in their central cavity [see Fig. 1(b)]. They are found in cells of plants and animals, where they are responsible for a variety of vital functions. Chen et al. show that the porphyrin has a different fluorescence spectrum, depending on the part of the molecule that is excited. Thanks to the highly localized tunneling current, the luminescence induced by the STM leads to a spectacular improvement in the spatial resolution of single-

DOI: $10.1103 /$ Physics.3.97

URL: http://link.aps.org/doi/10.1103/Physics.3.97

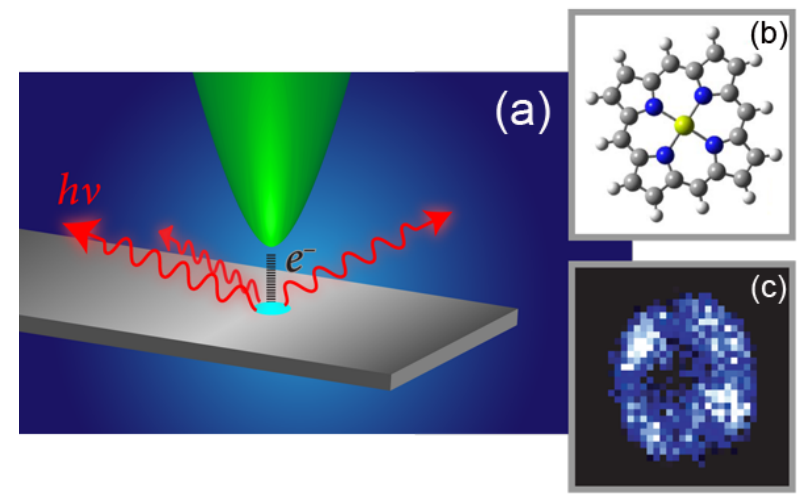

FIG. 1: (a) STM-induced luminescence process from an individual molecule. Under appropriate tunneling conditions the electrons excite the molecule, which relax via a radiative transition (fluorescence). (b) Molecular structure of a Mgporphyrin. (c) Photon map of a Mg-porphyrin. (Credit: Alan Stonebraker)

molecule optical microscopy.

In 1971, Russell Young-the inventor of the topographiner, a precursor of the STM-proposed to collect both secondary electrons and photons emitted from a tip-sample junction biased in the field-emission regime [5]. In 1981, Gerd Binnig and Heinrich Rohrer invented the STM, which is based on the quantum mechanical tunneling effect that leads to a current flow between a tip and a sample brought to within a nanometer of each other and biased by a few volts [6]. The STM can image metallic or semiconducting surfaces and nanostructures with atomic resolution. Soon after the STM's invention, reports that light could be emitted from a STM junction appeared. This phenomenon occurs in a variety of systems $[7,8]$ and can be classified into two categories according to the physical origin of the light emission: an

(C) 2010 American Physical Society 
excitation of a surface plasmon that emits light as it decays, or another process, where the current excites a system with well-defined energy levels (such as a molecule or quantum dot) that then fluoresces as it returns to its ground state.

This technique-referred to as STM-induced luminescence or STM-induced light emission-provides atomicscale optical spectroscopic information. The precise resolution originates from the highly local character of the excitation source (the tunneling electrons), which makes it possible to select and characterize single objects at the nanoscale. Several measurement techniques are possible, among them, total light intensity imaging (often simultaneously recorded with topography), spectroscopic mapping (where only a specific wavelength window is selected), single-point spectroscopy (light intensity versus wavelength), or yield measurements (photon intensity versus applied voltage).

A few years ago, Wilson Ho, an author of the new paper, led his experimental group to demonstrate the power of this technique using the STM to obtain fluorescence spectra from single porphyrin molecules [9]. In that experiment, porphyrins were thermally evaporated onto a cold $(10 \mathrm{~K})$, partially oxidized NiAl surface, producing a sample in which the molecules were isolated from each other. Different oxide adsorption sites and geometries led to different molecular conformations at the surface, which subsequently affected the lack or the presence of luminescence. The researchers observed variations in the light emission spectra from different parts of the molecule, demonstrating that excitation, relaxation, and emission take place at the submolecular scale.

In their paper [4], Chen et al. go farther, combining topographic imaging, electronic and optical spectroscopy, and photon mapping to characterize the luminescence of single molecules with submolecular resolution. The first step consists of simultaneous acquisition of a standard STM topographic image and a photon map of phorphyrin molecules deposited on a thin oxide layer grown on a metal surface. The net emitted light, i.e., the signal integrated over all wavelengths, is acquired using an avalanche photodiode, a very sensitive semiconductor device that converts light into an electric signal. Some molecules do not emit light because of their conformation. Luminescent porphyrins appear as slightly elongated protrusions in the STM images, while in the photon maps they show up as ringlike patterns, with four bright maxima and a dark center. Comparing the measurements to the porphyrin geometry, the authors deduce that the optical transitions occur at the molecular macrocycle formed by the four pyrroles; the central cavity, where the metal atom (magnesium in this case) sits, is not involved in the luminescence process [see Fig. 1(c)]. This procedure enables Chen and coauthors to achieve the spatial imaging of these fluorophores with submolecular resolution.

The next step is the spectral resolution of the lu- minescence. The authors determine the fluorescence spectra (light intensity versus wavelength) at different parts of the molecule by performing single-point spectroscopy. The four bright maxima forming the ring pattern in the photon maps are not equivalent, but they can be grouped in pairs (equivalent maxima are found at the opposite sites of the ring). This observation agrees with STM imagery-the emitting molecules present a twofold symmetry (the elongated shape) instead of the circular (fourfold) symmetry characteristic of the freestanding porphyrins. Therefore it is reasonable to deduce that the molecules undergo a structural deformation, which reduces their symmetry. The two types of luminescence spectra from nonequivalent regions of the macrocycle differ significantly, but the most evident characteristic is that the main threshold peak shows up at slightly different wavelengths (about $762 \mathrm{~nm}$ for one type, and about $772 \mathrm{~nm}$ for the other type). Selecting a narrow wavelength window around the main peak of a spectrum, the authors construct a spectrally resolved map of the luminescence of the porphyrin molecule.

Chen et al. propose that the observed luminescence originates from the transition between the lowest unoccupied (LUMO) and the highest occupied (HOMO) molecular orbital. The latter is shifted above the Fermi level by the electric field induced by the applied voltage. The LUMO of porphyrins consists of two orthogonal orbitals, which are degenerate for freestanding molecules. However, this degeneracy is lifted for distorted porphyrins adsorbed on the oxide surface. As a consequence, the two LUMO states can have different vibronic couplings with the HOMO orbital, leading to the difference observed in the luminescence spectra and to the submolecular contrast in the spectral maps.

STM-induced luminescence spectroscopy and microscopy combined with STM imaging and tunneling spectroscopy constitutes a very complete analytical tool to investigate and characterize nanoscale objects, as demonstrated by Chen and co-workers' work on single porphyrin molecules [4]. These experiments are very demanding, but these combined methods improve our comprehension of fundamental processes taking place at the nanometer scale.

\section{References}

[1] Frontiers in Chemistry: Single Molecules, Special Issue, Science 283 (1999).

[2] A. A. Deniz, S. Mukhopadhyay, and E. A. Lemke, J. R. Soc. Interface 5,15 (2008).

[3] B.-S. Yeo, J. Stadler, T. Schmid, R. Zenobi, and W. H. Zhang, Chem. Phys. Lett. 472, 1 (2009).

[4] C. Chen, P. Chu, C. A. Bobisch, D. L. Mills, and W. Ho, Phys. Rev. Lett. 105, 217402 (2010).

[5] R. D. Young, Phys. Today 24, No. 11, 42 (1971).

[6] G. Binnig, H. Rohrer, Ch. Gerber, and E. Weibel, Phys. Rev. Lett. 49, 57 (1982).

[7] R. Berndt, in Scanning Probe Microscopy: Analytical Methods, edited by R. Wiesendanger (Springer-Verlag, Berlin, 1998).

(C) 2010 American Physical Society 


\section{About the Author}

\section{Marina Pivetta}

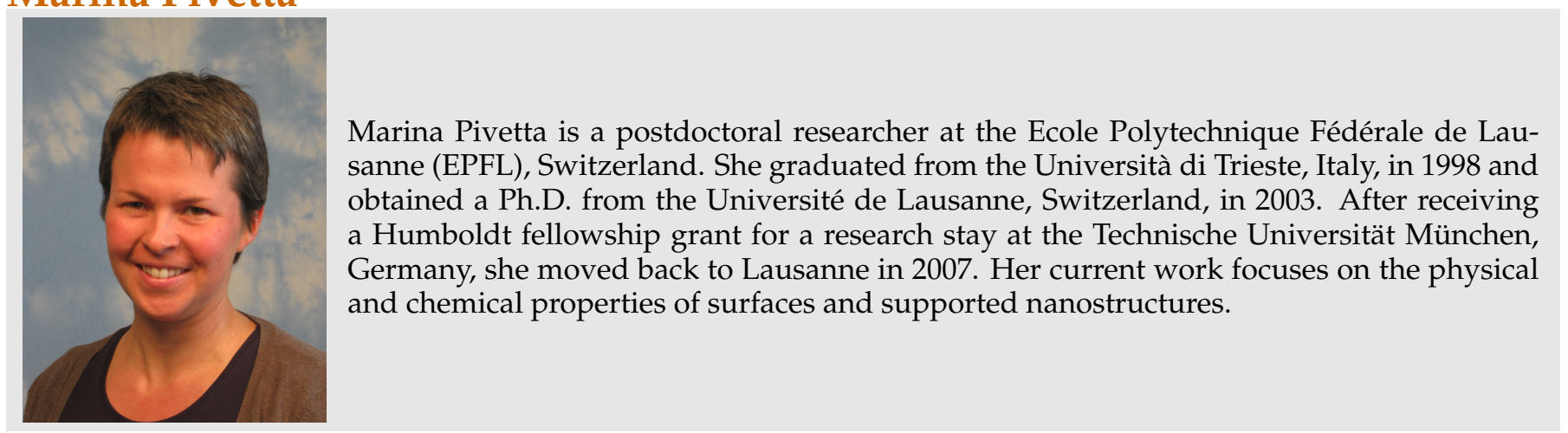

